\title{
THE MEASUREMENT OF BRANCHING RATIOS OF SPONTANEOOS RADIATIVE TRANSITION PROBABILITIES FOR Be-LIKE IONS N IV AND 0 V
}

J. Lang, R.A. Hardcastle and P.H. Spurrett Space and Astrophysics Division, Rutherford Appleton Laboratory, Chilton, Didcot, Oxfordshire OX11 OQX, UK

In an experiment to measure emission line intensities from the $\mathrm{Be}-$ like ion $\mathrm{Ne} V I I$ (Lang 1983), the intensity ratio $I\left(2 \mathrm{~s}^{3} \mathrm{~s}^{3} \mathrm{~s}-2 \mathrm{~s} 3 \mathrm{p}^{3} \mathrm{P}\right) /$ $I\left(2 p^{2} \quad 3 p-2 s 3 p^{3} p\right)$ was found to be a factor six larger than predicted from the ratio of the theoretical spontaneous radiative transition probabilities (A values). Indeed, to reconcile the Ne VII experiment and theory in several respects it was suggested also that in Be-like C III the theoretical ratios $I\left(2 s^{1} s^{1} s-2 s 3 p^{1} P\right) / I\left(2 p^{2}{ }^{1} D-2 s 3 p^{1} P\right)$ was about a factor three too low.

The same apparatus was used to measure A value ratios in Be-like $\mathrm{N}$ IV and $0 \mathrm{~V}$. Briefly the $40 \mathrm{~kJ} \theta$-pinch plasma source was viewed radially using a $1 \frac{1}{2} \mathrm{~m}$ Ebert and a $1 \mathrm{~m}$ grazing incidence photoelectric spectrometer. The $1 \frac{1}{2} \mathrm{~m}$ Ebert was intensity calibrated from $7300 \AA$ to $3600 \AA$ using a tungsten filament lamp, and from $3800 \AA$ to $2500 \AA$ using a deuterium lamp, both lamps having been calibrated by NPL. As described fully by Lang (1983), the branching-ratios method was used to calibrate the front exit slit of the grazing incidence instrument in the wavelength range 88 \& to $500 \AA$. Only Li-like line pairs of those listed in that paper were used with the addition of the $C$ IV doublets $2 s^{2} s-3 p^{2} P(312.4 A)$ and $3 s^{2} s-3 p^{2} P$ $(5801.33 \AA, 5811.98 \AA)$. The H-like ion He II $1-4$ transition $(243.0 \AA)$ and 3-4 transition $(4685.7 \AA)$ were used to cross-check the calibration. As shown in the figure, the He II result agrees excellently with the calibration as defined from the Li-like lines.

A comparison of the measured ratios with the theoretical ratios is given in the table. Theoretical ratios are the average of values obtained from the references numbered 1 to 6 and 9 to 11 . The uncertainty quoted includes both that from the measured ratios (typically $\pm 20 \%$ ) and the standard deviation of the mean of the theoretical values. The $2 s 2 p^{3} p-2 s 3 d^{3} D$ transitions are the most liable to be affected by opacity (1.e. radiation trapping) since they have high oscillator strengths and the majority of the ion population is in the $2 \mathrm{~s}^{2} \mathrm{p}^{3} \mathrm{p}$ metastable term (Keenan et al. 1984, Lang 1983). Experimental checks showed that the results quoted here are not affected by opacity.

Consider the first three rows of the table, involving transitions with a single electron jump. Two of the five ratios are unity to within the quoted uncertainty. The other 3 ratios differ from unity, in one case by a factor 2.3. Although the theoretical oscillator strengths (f) as given in the table are of the same order of magnitude, the A value for the 2-3 transition is approximately three orders of magnitude greater than that for the 3-3 transition. The 2-3 transition contributes $>90 \%$ of the total decays from the upper level. Thus, although the theoretical papers quote agreement between theoretical lifetimes and those measured by 
the beam foil method, the 3-3 transition $A$ values could well be causing the discrepancies found.

For measured ratios 1nvolving $2 \mathrm{p}^{2}-2 s 3 p$ transitions, the discrepancies between theory and experiment are much larger and are attributed to the theoretical A values for these transitions. The calculations are difficult as the transitions depend on configuration mixing for their existence and have small oscillator strengths. Perhaps experiments of this type are the best way of obtaining reliable $A$ values for such transitions.

\section{REFERENCES}

(1) Fawcett, B.C., 1984, Atomic Data and Nuclear Data Tables, 30, 1.

(2) Glass, R., 1979, J.Phys.B: At.Mol.Phys., 12, 1633.

(3) Glass, R., 1981, Z.Phys.A - Atoms and Nuclei, 299, 15.

(4) Hibbert, A., 1976, J.Phys.B: At.Mol.Phys., 9, 2805.

(5) Hibbert, A., 1980, J.Phys.B: At.Mol.Phys., 13, 1721.

(6) Hummer, D.G. and Norcross, D.W., 1974, Mon.Not.R.astr.Soc., $168,263$.

(7) Keenan, F.P., Berrington, K.A., Burke, P.G., Kingston, A.E. and Dufton, P.L., 1984, Mon.Not.R.astr.Soc., 207, 459.

(8) Lang, J., 1983, J.Phys.B: At.Mol.Phys., 16, 3907.

(9) Laughlin, C., Constantinides, E.R. and Victor, G.A., 1978, J.Phys.B: At.Mol.Phys., 11, 2243.

(10) Malinovsky, M., 1975, Astronomy \& Astrophysics, 43, 101.

(11) Nussbaumer, H., 1972, Astronomy \& Astrophysics, 16, 77. 


\section{RESULTS FOR EXPERIMENTAL BRANCHING-RATIO/ THEORETICAL BRANCBING-RATIO}

RATIO

$\frac{A\left(2 s^{3} s^{1} s-2 s^{3} p^{1} P\right)}{A\left(2 s^{2}-2-2 s^{3} p^{1} P\right)}$

$\frac{A\left(2 s^{3} p^{1} p-2 s^{3} d^{1} D\right)}{A\left(2 s^{2} p^{1} p-2 s 3 d^{1} D\right)}$

$\frac{A\left(2 s^{3} p^{3} p-2 s 3 d^{3} D\right)}{A\left(2 s 2 p^{3} p-2 s 3 d^{3} D\right)}$

$\frac{A\left(2 s^{3} s^{1} s-2 s^{3} p^{1} P\right)}{A\left(2 p^{2}{ }^{1} D-2 s^{3} p^{1} P\right)}$

$\frac{A\left(2 s 3 s^{3} s-2 s 3 p^{3} P\right)}{A\left(2 p^{2} 3 p-2 s 3 p^{3} P\right)}$
N IV

$-\quad 1.6 \pm 0.3$

$1.6 \pm 0.4$

$1.2 \pm 0.4$

$1.3 \pm 0.3$

$3.4 \pm 1.0$

$0.05 \pm 0.02$ $f(0 \quad v)$

0.214

0.389

0.210

0.561

0.116

0.644

0.214

0.020

0.576

0.0004

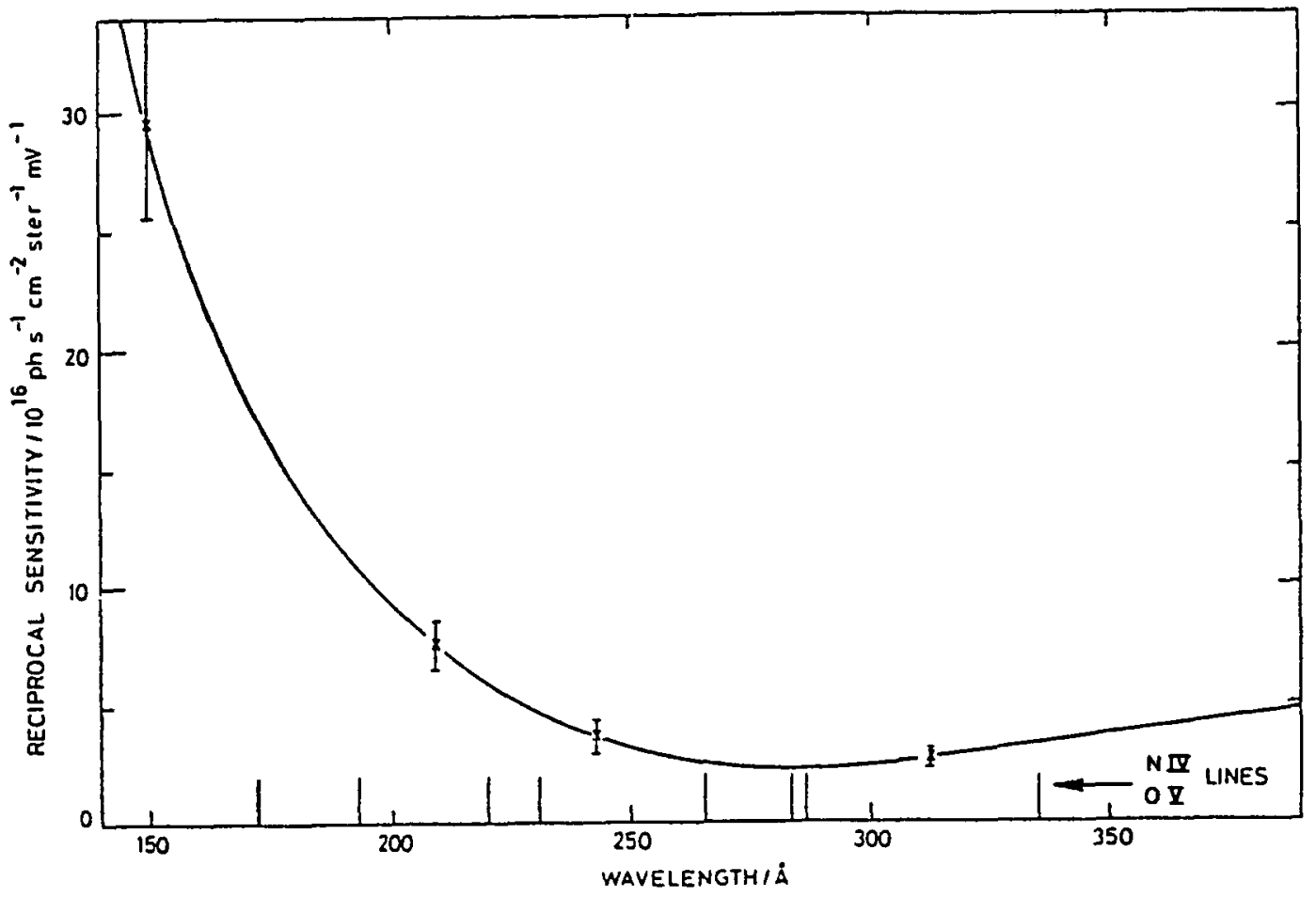

